\title{
Nerve Conduction Velocity Test
}

National Cancer Institute

\section{Source}

National Cancer Institute. Nerve Conduction Velocity Test. NCI Thesaurus. Code C88502.

A test that measures the speed of nerve activity along the length of a nerve. It is used to detect nerve damage or dysfunction. 